\title{
International student-athlete transition into collegiate sport in the United States
}

\section{BACKGROUND}

Researchers have explored international student-athletes' (ISA) transitions within the NCAA, often focusing on physical and psychological difficulties, barriers, coping, and influences on performance. The transition into US collegiate sport for ISA not only includes the typical obstacles associated with adapting to college, such as navigating socialization opportunities, experiences of homesickness, or interpreting differences in academic structure, but also those related to joining a new system within sport in an unfamiliar cultural setting. However, most of the published work has focused on barriers and problems. Investigations of positive elements in athlete career transition have been limited, yet are an important element in understanding how to support ISA.

\section{PARTICIPANTS AND PROCEDURE}

The current exploratory qualitative study examined the perceptions of the types of resources, training, and positive interventions reported to be most helpful for interna- tional student-athletes transitioning into collegiate sport in the United States. Five NCAA Division I athletes (three females and two males, from 4 different countries) and two NCAA Division I male coaches participated in individual in-depth interviews.

\section{RESULTS}

The central themes that emerged focused on: supportive resources, coping processes, adjustment to team, and adjustment to culture.

\section{CONCLUSIONS}

These results have implications for understanding effective responses to the obstacles that arise for ISA, as well as purposeful implementation of strategies to help athletes adjust during what is typically a challenging transition period.

\section{KEY WORDS}

coping; athletic career transition; international studentathletes; supportive resources; adjustment to college

ORganization - 1: University of Denver, Denver, CO, United States · 2: University of Colorado Anschutz Medical Campus, Aurora, CO, United States; University of Colorado Boulder, Boulder, CO, United States · 3: University of Wyoming,

Laramie, WY, United States

AUthors' Contributions - A: Study design - B: Data collection - C: Statistical analysis - D: Data interpretation .

E: Manuscript preparation · F: Literature search · G: Funds collection

Corresponding aUthor - Melissa G. Streno, Psy.D., University of Denver, 2199 S University Blvd., Denver, CO 80208,

United States, e-mail: melissa.streno@gmail.com

to Cite this ARticle - Streno, M. G., Poczwardowski, A., Welton-Mitchell, C., \& Moody, E. (2020). International studentathlete transition into collegiate sport in the United States. Health Psychology Report, 8(1), 38-46. https://doi.org/ 10.5114/hpr.2019.90383

RECEIVED 12.09.2019 • REVIEWED 10.10.2019 • ACCEPTED 09.11.2019 • PUBLISHED 05.12.2019 


\section{BACKGROUND}

Recent research has attempted to map out experiences of international student-athletes transitioning to collegiate athletics in the US (e.g., Lee \& Opio, 2011; Popp, Love, Kim, \& Hums, 2010). For example, Popp, Hums, and Greenwell (2009) found that NCAA student athletes from the US adjusted better socially and formed more solid attachments to their university compared to international student-athletes (ISA). However, most of the published work has focused on barriers and problems. Investigations of positive elements in athlete career transition have been limited, yet are an important element in understanding how to support ISA. North American Division I collegiate athletic programs present student-athletes with numerous challenges, but also provide opportunities for personal and athletic growth. Consequently, this study focuses on understanding what enables ISA to thrive during the period of transition to a new team, while simultaneously adapting to an unfamiliar cultural context.

Researchers have investigated athletic career transitions, identifying common physical and psychological difficulties, barriers, coping and influences on both adaptation and performance (e.g., Lee \& Opio, 2011; Pierce, Popp, \& Meadows, 2011; Stambulova, Franck, \& Weibull, 2012; Richards, 2014; Morris, Tod, \& Oliver, 2015). There is limited research, however, on the challenges facing ISA relocating to the US for college and entering high performing athletic environments. The transition into US collegiate sport for ISA not only includes the typical obstacles associated with adapting to college, such as navigating socialization opportunities, experiences of homesickness, or interpreting differences in academic structure, but also those related to joining a new system within sport in an unfamiliar cultural setting. Awareness of potential hindrances, such as communication barriers, role ambiguity, and unfamiliar norms, is essential in order to assertively take action to meet the needs of ISA (Bruner, Munroe-Chandler, \& Spink, 2008; Popp, 2007; Pierce et al., 2011). Additionally, coping skills, psycho-social interventions, phases in adaptation, and other approaches to resource development for athlete transitions to advanced training centers have received increasing attention from sport psychologists (e.g., Poczwardowski, Diehl, O’Neil, Cote, \& Haberl, 2014; Stambulova et al., 2012; Stambulova, Pehrson, \& Olsson, 2017).

Stambulova's six-stage athletic career transition model played an instrumental role in understanding various transitions in sport and included: (a) the beginning of sport specialization, (b) the transition to more intensive training in the chosen sport, (c) the transition from junior to senior/high achievement sport, (d) from amateur to professional sports, (e) from peak to the final stage, and (f) the transition to the post-career (Stambulova, 2003; Stambulova, Alfermann, Statler, \& Côté, 2009). Stambulova and colleagues $(2007,2009)$ describe a transition as a developmental shift, which may present either positive or negative outcomes and will require a method of coping. Such transitions can be experienced as pressure and are often associated with ambiguity about anticipated changes, as well as concerns about additional roles and responsibilities associated with life as a student-athlete. Additionally, Wylleman and Lavallee's (2004) developmental model emphasizes developmental obstacles encountered during the course of athletic career transitions, including transitions in academic, social and psychological realms.

In research on ISA, Lee and Opio (2011) examined the experience of 16 student-athletes from seven different African countries as they adapted to U.S. life. The findings stressed the need for coaches, teammates, and athletic personnel to understand ISA cultural backgrounds, their desire to learn and grow, and a realistic appraisal of their academic abilities. The difference in personal values between international and domestic student-athletes is often unnoticed and consequently not addressed during the transition. Lee and Opio also indicated distinctions in academic pressures and lifestyle changes between home and host countries. Further, the arrival point of ISA is the team; thus, establishing and promoting clear and consistent team communication (e.g., overcoming role ambiguity and promoting agreement) is fundamental to preventing breakdowns in team cohesion and performance (Cunningham \& Eys, 2007).

Coaches play a significant role in establishing team culture, promoting effective lines of communication, and building and maintaining team cohesion; thus, they need to embark on yet another role in the ISA transition to their team. Research suggests that, rather than ISA being viewed as the instigators of change or needing to conform to the new system, a more reasonable expectation is for all university athletic personnel to be involved in supporting the adjustment process. For instance, results from Lee and Rice's (2007) qualitative study indicated that some ISA encountered an "unwelcoming environment," undermining the potential for successful adjustment (Lee \& Opio, 2011, p. 634). This underscores the importance for coaching staff and team personal to create a hospitable environment, including awareness of added pressures that may occur for ISA beyond the normal challenges all college students face (Bruner et al., 2008).

As previously established, in order to help an individual cope, adjust, and succeed during athletic career transitions, there must be a stable and purposeful support system in place (e.g., Poczwardowski et al., 2014; Stambulova et al., 2012), especially considering the challenges and demands associated with being an ISA (e.g., Lee \& Opio, 2011). Pierce et al. (2011) called
International student-athlete transition 
Melissa G. Streno,

Artur

Poczwardowski, Courtney Welton-Mitchell, Eric Moody for more research on ISA transitions, especially with regard to their perception of the available (or not) social support. Thus, the purpose of this study is to address gaps in the literature through exploration of ISA perceptions and experiences related to adaptation to US Division I College Athletics, including identifying preferred supports to help ease transitions. A qualitative research design was used with the phenomenological analytic component (see Patton, 2002 for a description) to understand the participants' experiences, perceptions, and meanings.

\section{PARTICIPANTS AND PROCEDURE}

\section{PARTICIPANTS}

After Institutional Review Board (IRB) approval was granted, a purposeful sampling procedure (Patton, 2002) was used to select five ISA and two coaches from an NCAA Division I university to partake in this study (see Table 1). In particular, the benefit of interviewing student-athletes who were sophomore or junior year was to gain insight from those who have already experienced adjustment into US collegiate sport, and yet had fresh experiential and narrative access to this recent transition. The specific sport is not disclosed with the intention of protecting the participants' identity.

\section{INTERVIEW GUIDE}

An interview guide was used to ensure systematic and comprehensive data collection from all ISA participants and coaches. Interview questions incorporated Stambulova's perceived coping resources and Athletic Career Transition Model (1994, 2003), as well as Wylleman \& Lavallee's Developmental Model (2004). Topic areas included previous transitions, pre- transition experiences (after the decision to move to the US was made), and the expected transitional barriers an ISA might encounter when moving to the US to compete for their university. Questions regarding resources and support involving coaches, trainers, and other team personnel were included in both the ISA and coach interview guides. The ISA interview guide consisted of four sections: (a) opening and rapport building questions (e.g., "What were some of the important factors in your decision-making process for college?"); (b) introductory questions (e.g., "What was the contact, communication, and preparation prior to arrival in the US like (with coaches, teammates, academic counselors, etc.)?"); (c) support during transition (e.g., "In your opinion, what were the most supportive and accommodating elements during your transition?"); (d) ending reflection questions (e.g., "What could have been changed in order to make the adjustment easier, both prior to and during the transition?").

\section{DATA ANALYSIS}

We followed Creswell's (1998) spiral model of data analysis, which was consistent with those utilized in previous athletic transition research studies (e.g., Bruner et al., 2008; Schinke, Michel, Gauthier, Pickard, \& Danielson, 2006). Interviews were transcribed and coded by the first author. Using the qualitative data analysis research software program, ATLAS.ti, "core consistencies and meanings" within each interview were identified (Patton, 2002, p. 453). Specifically, key words and phrases (data points) were given a code. These codes were later used to configure emerging themes and categories in the final integration and interpretation of the findings (Creswell, 1998). Themes were viewed as descriptive findings (less abstract), while categories were defined as topical (more abstract). Through the processes of convergence and

Table 1

Participant demographics $(n=7)$

\begin{tabular}{lccccccc}
\hline Participant & Role & Gender & $\begin{array}{c}\text { Academic year/age } \\
\text { (years) }\end{array}$ & $\begin{array}{c}\text { Transfer } \\
\text { status }\end{array}$ & $\begin{array}{c}\text { Country } \\
\text { of origin }\end{array}$ & $\begin{array}{c}\text { Years } \\
\text { in US }\end{array}$ & $\begin{array}{c}\text { Years } \\
\text { in sport }\end{array}$ \\
\hline 1 & ISA & Female & Sophomore/19 & No & Wales & 1.5 & 11 \\
2 & ISA & Female & Sophomore/19 & Yes* & Belgium & 2 & 13 \\
3 & ISA & Male & Sophomore/20 & No & Portugal & 2 & 14 \\
4 & ISA & Male & Sophomore/19 & No & England & 2 & 12 \\
5 & ISA & Female & Junior/20 & No & England & 2.5 & 12 \\
6 & Coach & Male & - & - & USA & +15 & +15 \\
7 & Coach & Male & - & - & USA & +15 & +15 \\
\hline
\end{tabular}

Note. ${ }^{*}$ Participant 2 reported transferring to the university one year ago. 
divergence, repetitive regularities and differences amongst the findings were evaluated and incorporated into the discussion of the results (Patton, 2002).

Addressing 'trustworthiness' of the study required incorporating four essential components: integrity of the data, balance between objectivity and subjectivity, coherent communication of the results, and member checking (Williams \& Morrow, 2009; Lincoln \& Guba, 1985). To minimize the potential for misunderstanding due to language barriers (although fluency in English or participation in the English as a Second Language Program is required by U.S. universities), a high level of multicultural awareness was consistently applied. Nevertheless, during each interview clarification of responses to address any potential miscommunication due to language barriers was routinely used. Member checking was performed with four participants to verify the data generated from the participants, specifically "analytic categories, interpretations, and conclusions" (Lincoln \& Guba, 1985, p. 314). Finally, a study audit (the second author) was incorporated for assistance in challenging and verifying findings (Patton, 2002).

\section{RESULTS}

Four themes emerged capturing the perceptions and experiences of ISA, including the types of resources and interventions most helpful for ISA transitioning into collegiate sport in the United States: (a) supportive transition resources, (b) coping processes, (c) challenges in adjustment to the team, (d) challenges in adjustment to the culture (see Table 2).

\section{SUPPORTIVE TRANSITION RESOURCES}

Supportive transition resources referred to the variety of available resources present throughout the ISA transition to the U.S. culture and university system. Stambulova and colleagues define these as "all internal and external factors that facilitate the coping process" (2009, p. 399).

Preparation prior to transition. ISA responses highlighted the importance of previous experiences, such as a number of years participating in sport, previous travel to the US, and communication with prospective coaches, as influencing their current transition process and outcomes. Excitement about being a student-athlete in the U.S. and the opportunity to improve in sport, while simultaneously earning a degree, were often cited as considerations in the decision-making process.

Most ISA indicated they were eager to join a team, meet new friends, and gain independence, although many acknowledged that they did not have comparable experiences from which to make predictions about how things would unfold. Overall, there was a collective impression that the experience would be more relaxed than it actually was, specifically having less study, traveling, and training. Respondents noted fears about the transition related to leaving family, academic difficulties, likability of their team and coach, and other aspects of adapting to the US college experience, such as: "I was very scared about how I was going to feel about being away from my family" and "...writing college papers and talking in a class in front of a college professor, that was another fear I had".

Coach support. All ISA participants described consistent coach contact as a common occurrence that increased their comfort level with coaches. Described as "family," ISA indicated that coaches helped them feel more prepared, motivated, and less nervous, which ultimately helped performance. Coaches' willingness to connect them with academic personnel was also viewed as helpful and supportive.

Table 2

Themes capturing ISA perceptions and experiences

\begin{tabular}{|c|c|}
\hline $\begin{array}{l}\text { Higher-order } \\
\text { themes }\end{array}$ & Lower-order themes \\
\hline \multirow{8}{*}{$\begin{array}{l}\text { Supportive } \\
\text { transition } \\
\text { resources }\end{array}$} & Preparation prior to transition \\
\hline & Coaches' support \\
\hline & Contact prior to transition \\
\hline & Support around asking for help \\
\hline & $\begin{array}{l}\text { Family and friend contact/ } \\
\text { support }\end{array}$ \\
\hline & Helpful during transition \\
\hline & Suggestions for easier adjustment \\
\hline & Adjustment to academic life \\
\hline \multirow{2}{*}{$\begin{array}{l}\text { Coping } \\
\text { process }\end{array}$} & Coping strategies \\
\hline & $\begin{array}{l}\text { Extracurricular activities outside } \\
\text { sport }\end{array}$ \\
\hline \multirow[t]{4}{*}{$\begin{array}{l}\text { Adjustment } \\
\text { to the team }\end{array}$} & $\begin{array}{l}\text { Changes playing for a team } \\
\text { vs. as an individual }\end{array}$ \\
\hline & Handling conflict \\
\hline & $\begin{array}{l}\text { Realization about transition } \\
\text { to student-athlete }\end{array}$ \\
\hline & Team cohesion outside sport \\
\hline \multirow{4}{*}{$\begin{array}{l}\text { Adjustment } \\
\text { to the } \\
\text { culture }\end{array}$} & Cultural inquiry \\
\hline & Cultural differences \\
\hline & Language \\
\hline & Environmental differences \\
\hline
\end{tabular}

International student-athlete transition 
Melissa G. Streno,

Artur

Poczwardowski, Courtney Welton-Mitchell, Eric Moody
Coaches described their initial role in the ISA transition as a "caretaker" and "sounding board" which they hoped helped convey understanding of the challenges faced, as well as recognition of the differences facing each athlete. Among ISA, the impression of the support they received from coaches mirrors the coaches' emphasis on trying to make ISA feel safe, respected, and comfortable. Coaches further clarified that in order for an ISA to feel comfortable soliciting assistance through other campus resources (e.g., a counseling center), establishing a positive team culture was important.

Contact prior to transition. ISA shared gratitude for frequent communication with their coach and team. Those who visited the campus as part of their decision-making said that developing a sense of comfort with the coach was useful. Further, throughout the recruitment process and prior to the transition, ISA spoke with compliance and academic advisors, friends currently competing in the U.S., and professional liaisons. All ISA viewed these connections as opportunities to develop a better understanding of the collegiate lifestyle and U.S. culture.

Support around asking for help. ISA indicated that time spent with their team, coach, and trainers in their new environment gradually improved their confidence and comfort asking for help and understanding how Americans "went about stuff". All ISA noted constant availability of support, which helped to prevent failure in their new environment.

Family and friend contact and support. All ISA agreed that time differences and busy schedules made it difficult to communicate with family and friends back home. Most made attempts to talk to family members daily and appreciated having holiday breaks to travel home, especially considering financial limitations preventing family from visiting them regularly. Families were depicted as helpful and encouraging throughout the entire transition.

Helpful resources during transition. A welcoming and sensitive team setting, as well as time spent learning from teammates, most specifically seniors and other ISA, was identified as most comforting by ISA. Likewise, coaches viewed initial team bonding activities, which were intended to make the ISA feel included and cared for, as essential in order for a "family-like" environment to emerge. With regard to campus resources, the international house/office, international professors, and foreign languagespeaking graduate students, were viewed as useful and supportive during the transition.

Suggestions for easier adjustment. To facilitate adjustment, ISA requested earlier preparation, information, and more time spent with their team. Also, the one-on-one, in-person communication with coaches was important for learning about each other's backgrounds and personalities. Additional requests included more insight and warning about the intensity of academics, more time spent discussing practice expectations, and continual recruitment of additional international teammates.

Adjustment to academic life. Many respondents agreed that the U.S. university system is vastly different from what they were expecting. The difficulty with unfamiliar and required subjects was buffered by accessible tutors who were described as preferred resources for help.

\section{COPING PROCESS}

The coping process, which Stambulova describes as "central" (2009, p. 399) or vital throughout a transition, includes strategies and resources that align with transitional demands and help facilitate adaptation.

Coping strategies. ISA coped psychologically by staying "mentally tough", attempting to worry less about others' perceptions, remember personal goals, develop independence, allow for extra time, prepare in advance for realistic obstacles, and reach out for support around organization of academic responsibilities. Homesickness was managed by keeping family and country of origin in mind, staying busy, and approaching the transition one day at a time. Coaches indicated that previous experiences with ISA have made it easier for them to identify warning signs of homesickness, encourage useful coping strategies, and create a family-like environment.

Extracurricular activities outside of sport. There was a distinction between ISA who lacked sufficient time to build non-athlete acquaintances and those who made friends outside of sport. A few ISA also acknowledged that non-athletes' curiosity is often a conversation starter that develops into future relationships. Free time spent away from sport included a variety of active and social engagements, such as going to movies or exploring the city.

\section{ADJUSTING TO THE TEAM}

Adjusting to the team involves unfamiliar encounters, changes in routine, and in interpersonal dynamics, experienced by ISA, who typically lack previous team experience in sport.

Changes playing for a team versus as an individual. Unlike their American teammates, all participants had minimal previous team experience. The transition from an independent athlete to functioning within a team was challenging, overwhelming and tense because they were no longer representing just themselves. However, being part of a team eventually became helpful during their transition. Staying organized and following stricter rules were described as important in order to remain aware of their role and good preparation for life beyond sport. Most par- 
ticipants described seniors as caretakers with experience and ability to motivate and organize.

Handling conflict. ISA stated that conflict was previously addressed one-on-one. After joining their new team, ISA reported that coaches and team members consistently encouraged open discussion about feelings and issues with the team in a group format. Coaches' thoughts paralleled this idea and reiterated the importance of modeling open lines of communication and reminders of what "being a good teammate" entails.

Realizations about transition to student-athlete role. All participants spoke about the intensity and demands of being a student-athlete, describing the high level of sport and time required for practice and travel as "shocking." Team travel was a new experience for most, but provided support and helped reduce tension as ISA were learning to navigate the studentathlete balance. Being young and lacking familiarity with the U.S. were noted as factors contributing to difficulties, but for some, hopes and expectations were reached or exceeded after one year.

Team cohesion outside sport. With the coaches' encouragement for time spent as a team outside official team activities, ISA endorsed establishment of a "family-like" bond. While enduring challenges together increased tension, it also provided opportunities to constructively work through conflict, increase cohesion, and practice honesty without fearing judgment. ISA agreed that time together improved comfort within interpersonal relationships and performance.

Coaches described turning an individual sport into a team environment as one of their biggest challenges. However, they also acknowledged their position in helping foster team cohesion and time spent together outside sport. Strengthening this view, they added that by promoting team bonding, they were also helping athletes develop leadership qualities, communication skills, and increased comfort expressing themselves in a group setting.

\section{ADJUSTING TO THE CULTURE}

Adjusting to the culture encompassed multiple essential factors to be aware of throughout the ISA transition to US college sport.

Cultural inquiry. Participants agreed that coaches, teammates, and trainers consistently expressed interest in their backgrounds by politely and genuinely asking questions about their cultural lifestyles. Additionally, the coaches intentionally worked towards developing an awareness of cultural differences by sharing real-world examples and their own personal experiences. Icebreakers, team bonding exercises, and process groups for all ISA were facilitated with the intention of expanding their support networks with various teams throughout the university. More frequent team discussion, involving both ISA and Americans, was requested as another available outlet to process, educate, and provide clarity around the use of language.

Cultural differences. No responses indicated problems with acceptance from teammates or coaches, as well as any discriminatory incidents. A no tolerance policy for racist or derogatory comments was pervasive amongst the perceptions of participants, and if cultural comments were made, it was in a joking and inclusive manner. While diversity and inquiry about each other's differences were encouraged, ISA connected more with their international teammates. Groups that initially formed based on culture balanced out as teammates got to know one another.

ISA described food selection, tipping, using hands to eat, and dorm-style meals as challenging parts of the transition because of the differences from their home countries. Technology, as well as coaches' acceptance of their food choices, were encouraging and supportive during the adjustment: the role coaches play in building a culture of tolerance for multiculturalism and diversity was again paramount.

Language. The impact of language differences and accents varied amongst participants. While some ISA said Americans appeared lost in conversation because of pace and accent, others said their prior experience speaking English minimized the difficulty. Practicing English was challenging, yet previous English courses in high school and help from teammates with grammar and writing was helpful.

Environmental differences, including climate and weather, transportation, and city life were described as important elements for their acclimation in an unfamiliar location.

\section{DISCUSSION}

In response to previous research highlighting the challenges and obstacles faced by ISA adapting to US college sport and culture, this study explored the ISA perceptions and experiences related to both the adaptation to US Division I College Athletics, as well as supportive strategies established to ease their transitions. The results illustrate the value of developing greater awareness for effective strategies, as well as purposeful implementation of interventions, for ISA during their period of adjustment to the US.

Our findings suggested that contact established by the coach and current team members prior to the transition was extremely valuable because it helped establish relationships, foster a welcoming environment, and provide beneficial knowledge about the new environment. Additionally, a supportive foundation of university administration, support staff, and international campus resources, was described to have helped the ISA balance developing autono-
International student-athlete transition 
Melissa G. Streno,

Artur

Poczwardowski, Courtney

Welton-Mitchell, Eric Moody my with comfort required to ask for help. While responses closely aligned with past research findings suggesting the importance of consistent communication patterns and attempts at gaining knowledge of ISA backgrounds early in the transition (Cunningham \& Eys, 2007; Lee \& Opio, 2011), the data in our study indicated that contact prior to the transition was also extremely valuable, especially interactions and relationships established early on. More organized and concrete ways of connecting ISA with other international campus resources, groups, or other personnel in helping roles could be beneficial in reaching a higher number of ISA. The suggestion for an ISA-specific orientation, coupled with the ISA expressed appreciation for more individual attention, may also provide a more solid foundation during their transition. These necessary interventions relate to and support Lee and Rice's (2007) research advocating the development of increased initiative and participation from the university in the ISA adaptation and the need for efforts to reinforce ongoing contact with family and friends.

As Stambulova and colleagues $(2007,2009)$ pointed out, various outcomes of a transition require coping strategies. This was a crucial consideration in this study considering that none of the ISA reported previous mental skills training or sport psychology experience. Based on the results from our study, one response to Bruner et al.'s (2008) request for qualitative research directly considering specific supportive factors is that coping psychologically involves consistent practice of independence, focus on the controllables, support from others, and academic organization. Both the athletic career transition model (Stambulova, 2003) and the human adaptation to a transition model (Schlossberg, 1981) provide clear direction and insight regarding coping strategies needed to support ISA throughout their adjustment. Specifically, our results confirm the need for understanding the environment prior to a transition, available resources, transitional demands, and coping skills that either assist or hinder the success of the adaptation (Stambulova, Stephan, \& Japhag, 2007).

While previous research studies have demonstrated both instances of ISA being better prepared for the academic demands of college than Americans (Ridinger \& Pastore, 2000; Bale, 1987, as cited in Popp et al., 2009), as well as experiencing academic challenges and lower levels of motivation (Bruner et al., 2008; Lee \& Opio, 2011; Wylleman \& Lavallee, 2004; Pierce et al., 2011), our study aligned with findings that balancing study with daily obligations to sport is an obstacle experienced by ISA during their transition.

As called for by previous research (Pierce et al., 2011; Lee \& Opio, 2011; Bruner et al., 2008), our study demonstrates that there are effective resources and interventions being used by coaches and athletes. Enforcing certain expectations, such as organization, timeliness, and senior leadership, served as influential prompts for ISA regarding individual roles within the team. This support helped shift the mindset from the individual to the group and intensify their excitement for simultaneously pursuing a degree. Our findings also suggest that more time spent together during the transition could increase perceptions of well-being, reduce anxiety, and, as believed by the participants, improve team performance and success.

As requested previously, further insight concerning ISA cultural adjustment is necessary in order to implement effective and sensitive interventions (Lee \& Opio, 2011; Schinke et al., 2006). In our sample, practicing acceptance and tolerance for diversity by asking questions about one another's culture demonstrated genuine interest and immediate dismissal of racist remarks. This was also shown to help team cultural groupings dissolve as time progressed. Contrary to Lee and Opio's research (2011) indicating a felt sense of "indifference" from Americans concerning interest in ISA cultural backgrounds, our results consistently conveyed interest, awareness, and acknowledgement towards the ISA challenges as being most helpful.

\section{LIMITATIONS AND FUTURE DIRECTIONS}

The first limitation concerns the participants solicited from one individual sport. The small sample size does not represent shared adjustment experiences of ISA within NCAA Division I. Future research investigating various individual and team sports and ISA from a representative sample of geographical regions might provide more reliable insights into such transitions. This is important to capture the multicultural dynamics on how the host team receives ISA. Collectively, qualitative, quantitative, and mixed method designs (Giacobbi, Poczwardowski, \& Hager, 2005) will be able to address the complexities involved in ISA adapting to and thriving in American college sports.

Second, based on the common themes and perceptions of helpful support identified within the current study, a longitudinal study that purposefully implemented select recommended support strategies, interventions, or techniques, could propel future implementation of both standardized and program-focused interventions for ISA. Inclusion of a reliable and valid assessment tool, such as the Change-Event Inventory (Samuel \& Tenenbaum, 2011), could provide future insight and direction related to intervention outcomes. Similarly, the data obtained in this study exemplify the value of future system-level exploration about the role athletic department administrations have in training and developing competent coaches and athletic support staff in preparation for ISA acclimation to US culture and the collegiate system. 


\section{RECOMMENDATIONS FOR PRACTICE}

Results from our study might support several tentative recommendations for interventions, strategies, and approaches that, if cautiously and sensitively implemented, could help ISA adapt to US culture and feel supported through transitional obstacles. As early as possible, in-person contact between coaches, teammates, and prospective ISA, as well as on-campus visits, would be advisable. As another example, considering the experienced differences in academic structure between the US and other countries, specific attention should be given to preparing the ISA for that element of the transition, as well as strong encouragement for the use of tutors once in the US. In order to maintain and model an attitude of awareness and acknowledgement for the individual cultural differences amongst their team, the results suggest that a coach asking questions and showing genuine interest in the athlete's backgrounds demonstrates the significance of talking about diversity, even when it feels uncomfortable. It is essential to promote the utilization of resources to manage the unfamiliarity of a new culture; therefore, more specific orientation activities that would highlight multiculturalism should be encouraged. Finally, routinely spending time together has a potential to build team cohesion and help ISA acclimate to their daily routine.

In summary, the data from this study indicate the dynamic and multiphasic nature of international student-athletes' initial adapting to NCAA Division I academic and athletic experience. Based on the interview data, creating and utilizing supportive resources, investing into the coping process, and actively adjusting to team and culture seem to underlie positive transitions and additionally were perceived as effective strategies that originated from the coaches, athletic department, and the university. We anticipate that future research exploring the outcome of implementing these strategies with more diverse populations and sports, as well as the use of specific assessment tools, will continue to guide this movement towards making the transition for ISA more efficient and accommodating.

\section{REFERENCES}

Bruner, M. W., Munroe-Chandler, K. J., \& Spink, K. S. (2008). Entry into elite sport: a preliminary investigation into the transition experiences of rookie athletes. Journal of Applied Sport Psychology, 20, 236-252. https://doi.org/10.1080/10413200701867745

Creswell, J. (1998). Qualitative inquiry and research design: Choosing among five traditions. Thousand Oaks, CA: Sage Publications, Inc.

Cunningham, I. J., \& Eys, M. A. (2007). Role ambiguity and intra-team communication in interde- pendent sport teams. Journal of Applied Social Psychology, 37, 2220-2237. https://doi.org/10.1111/ j.1559-1816.2007.00256.x

Giacobbi, P. R., Poczwardowski, A., \& Hager, P. (2005). A pragmatic research philosophy for applied sport psychology. The Sport Psychologist, 19, 18-31. https://doi.org/10.1123/tsp.19.1.18

Lee, J., \& Opio, T. (2011). Coming to America: Challenges and difficulties faced by African studentathletes. Sport, Exercise, and Society, 16, 629-644. https://doi.org/10.1080/13573322.2011.601144

Lee, J. J., \& Rice, C. (2007). Welcome to America? International student perceptions of discrimination and neo-racism. Higher Education, 53, 381-409. https://doi.org/10.1007/s10734-005-4508-3

Lincoln, Y. S., \& Guba, E. G. (1985). Naturalistic inquiry. Newbury Park, CA: Sage Publications.

Morris, R., Tod, D., \& Oliver, E. (2015). An analysis of organizational structure and transition outcomes in the youth-to-senior professional soccer transition. Journal of Applied Sport Psychology, 27, 216234. https://doi.org/10.1080/10413200.2014.980015

Patton, M. Q. (2002). Qualitative research and evaluation methods ( $3^{\text {rd }}$ ed.). Thousand Oaks, CA: Sage Publications.

Pierce, D., Popp, N., \& Meadows, B. (2011). Qualitative analysis of international student-athlete perspectives on recruitment and transitioning into American college sport. The Sport Journal, 14, 1-14. Poczwardowski, A., Diehl, B., O’Neil, A., Cote, T., \& Haberl, P. (2014). Successful transitions to the Olympic Training Center, Colorado Springs: a mixed-method exploration with six resident athletes. Journal of Applied Sport Psychology, 26, 33-51. https://doi.org/10.1080/10413200.2013.773950

Popp, N. (2007). International student-athlete perception of college sport and its effect on adjustment to college. Electronic Theses and Dissertations, paper 1142. https://doi.org/10.18297/etd/1142

Popp, N., Hums, M. A., \& Greenwell, T. C. (2009). Do international student-athletes view the purpose of sport differently than United States studentathletes at NCAA Division I Universities? Journal of Issues in Intercollegiate Athletics, 2, 93-110.

Popp, N., Love, A. W., Kim, S., \& Hums, M. A. (2010). Cross-cultural adjustments and international collegiate athletes. Journal of Intercollegiate Sport, 3, 163-181.

Richards, V. (2014). International student-athlete: An acculturation effect (doctoral dissertation). Retrieved from http://search.proquest.com/docview/ 1617975427

Ridinger, L., \& Pastore, D. (2000). International student-athlete adjustment to college: a preliminary analysis. NACADA Journal, 20, 33-41. https://doi. org/10.12930/0271-9517-20.1.33

Samuel, R. D., \& Tenenbaum, G. (2011). The role of change in athletes' careers: a scheme of change for
International student-athlete transition 
Melissa G. Streno,

Artur

Poczwardowski,

Courtney

Welton-Mitchell,

Eric Moody sport psychology practice. The Sport Psychologist, 25, 233-252. https://doi.org/10.1123/tsp.25.2.233

Schinke, R. J., Michel, G., Gauthier, A. P., Pickard, P., \& Danielson, R. (2006). The adaptation to the mainstream in elite sport: a Canadian Aboriginal perspective. The Sport Psychologist, 20, 435-448. https://doi.org/10.1123/tsp.20.4.435

Schlossberg, N. K. (1981). A model for analyzing human adaptation to transition. The Counseling Psychologist, 9, 2-18. https://doi.org/10.1177/ 001100008100900202

Stambulova, N. (1994). Developmental sports career investigations in Russia: a post-perestroika analysis. The Sport Psychologist, 8, 221-237. https://doi. org/10.1123/tsp.8.3.221

Stambulova, N. (2003). Symptoms of a crisis-transition: a grounded theory study. In N. Hassmen (Ed.), SIPF Yearbook, 2003 (pp. 97-109). Orebro, Sweden: Orebro University Press.

Stambulova, N., Alfermann, D., Statler, T., \& Côté, J. (2009). Career development and transitions of athletes: the ISSP position stand. International Journal of Sport \& Exercise Psychology, 7, 395-412. https://doi.org/10.1080/1612197X.2009.9671916

Stambulova, N., Franck, A., \& Weibull, F. (2012) Assessment of the transition from junior-to-senior sports in Swedish athletes. International Journal of Sport and Exercise Psychology, 10, 79-95. https:// doi.org/10.1080/1612197X.2012.645136

Stambulova, N. B., Pehrson, S., \& Olsson, K. (2017). Phases in the junior-to-senior transition of Swedish ice hockey players: From a conceptual framework to an empirical model. International Journal of Sports Science \& Coaching, 12, 231-244. https:// doi.org/10.1177/1747954117694928

Stambulova, N., Stephan, Y., \& Japhag, U. (2007). Athletic retirement: a cross-national comparison of elite French and Swedish athletes. Psychology of Sport and Exercise, 8, 101-118. https://doi.org/10.1016/ j.psychsport.2006.05.002

Williams, E. N., \& Morrow, S. L. (2009). Achieving trustworthiness in qualitative research: a pan-paradigmatic perspective. Psychotherapy Research, 19, 576582. https://doi.org/10.1080/10503300802702113

Wylleman, P., \& Lavallee, D. (2004). A developmental perspective on transitions faced by athletes. In M. R. Weiss (Ed.), Developmental sport and exercise psychology: a lifespan perspective (pp. 503-524). Morgantown, WV: Fitness Information Technology. 